\title{
Effects of grape seed extract beverage on blood pressure and metabolic indices in individuals with pre-hypertension: a randomised, double-blinded, two-arm, parallel, placebo-controlled trial
}

\author{
Eunyoung Park ${ }^{1}$, Indika Edirisinghe ${ }^{1}$, Ying Yng Choy $^{2}$, Andrew Waterhouse ${ }^{2}$ and Britt Burton-Freeman ${ }^{1 *}$ \\ ${ }^{1}$ Center for Nutrition Research, Institute for Food Safety and Health, Illinois Institute of Technology, Chicago, IL 60616, USA \\ ${ }^{2}$ Department of Viticulture and Enology, University of California, Davis, CA 95616, USA
}

(Submitted 28 April 2015 - Final revision received 18 September 2015 - Accepted 5 October 2015 - First published online 16 November 2015)

\section{Abstract}

The aim of the present study was to test grape seed extract (GSE) as a functional ingredient to lower blood pressure (BP) in individuals with pre-hypertension. A single-centre, randomised, two-arm, double-blinded, placebo-controlled, 12-week, parallel study was conducted in middle-aged adults with pre-hypertension. A total of thirty-six subjects were randomised (1:1) to Placebo ( $n$ 18) or GSE ( $n$ 18) groups; twentynine of them completed all the protocol-specified procedures (Placebo, $n$ 17; GSE, $n$ 12). Subjects consumed a juice (167 kJ ( $40 \mathrm{kcal}$ )) containing $0 \mathrm{mg}$ (Placebo) or $300 \mathrm{mg} / \mathrm{d}$ GSE $(150 \mathrm{mg}$ ) twice daily for 6 weeks preceded by a 2-week Placebo run-in and followed by 4-week no-beverage follow-up. Compliance was monitored. BP was measured at screening, 0, 6 and 10 weeks of intervention and blood samples were collected at 0, 3, 6 and 10 weeks of intervention. GSE significantly reduced systolic BP (SBP) by $5 \cdot 6 \%(P=0 \cdot 012)$ and diastolic BP (DBP) by $4.7 \%(P=0.049)$ after 6 weeks of intervention period, which was significantly different (SBP; $P=0.03)$ or tended to be different (DBP; $P=0.08$ ) from Placebo. BP returned to baseline after the 4-week discontinuation period of GSE beverage. Subjects with higher initial BP experienced greater BP reduction; nearly double the effect size. Fasting insulin and insulin sensitivity tended to improve after 6 weeks of GSE beverage supplementation $(P=0.09$ and 0.07 , respectively); no significant changes were observed with fasting plasma lipids, glucose, oxidised LDL, flow-mediated dilation or vascular adhesion molecules. Total plasma phenolic acid concentrations were 1.6 times higher after 6 weeks of GSE $v$. Placebo. GSE was found to be safe and to improve BP in people with pre-hypertension, supporting the use of GSE as a functional ingredient in a low-energy beverage for BP control.

Key words: Grape seed extract: Blood pressure: Pre-hypertension: Endothelial function: Insulin sensitivity: Polyphenols

Hypertension is a major risk factor for fatal and non-fatal CVD events ${ }^{(1-3)}$. Hypertension may affect $>90 \%$ of individuals during their lifetimes ${ }^{(4)}$. Current estimates indicate that hypertension costs the USA almost $\$ 70$ billion annually in direct medical expenses ${ }^{(5)}$. Randomised clinical trials and meta-analyses indicate that blood pressure (BP) reductions lower the risk of myocardial infarction, stroke and other cardiovascular deaths ${ }^{(6,7)}$. Therefore, adequate control of BP in the healthy range is of enormous individual and public health importance.

Pre-hypertension is a condition defined by a systolic BP (SBP) between 120 and $139 \mathrm{mmHg}$ or a measured diastolic BP (DBP) between 80 and $89 \mathrm{mmHg}^{(8)}$. The Framingham Heart Study showed that the 4-year incidence of hypertension increased between 17.6 and $37.3 \%$ in individuals with pre-hypertension between 35 and 64 years of age ${ }^{(9)}$. Current medical practice does not treat pre-hypertension per se; however, the importance of lifestyle modifications are emphasised in the Joint National Committee (JNC)-7 and JNC-8, which recommend weight control, regular exercise and following a healthy diet ${ }^{(8,10,11)}$.

Diet plays a significant role in maintaining healthy $\mathrm{BP}^{(10)}$. In addition to the widely accepted attributes of the Dietary Approaches to Stop Hypertension eating plan, emerging evidence suggests that certain dietary factors may have BPlowering effects, thus warranting inclusion in the diet, particularly when a condition is becoming apparent, such as the warning sign of pre-hypertension. Certain polyphenolic compounds/compositions promote $\mathrm{BP}$ regulation and improve vascular health $^{(12)}$. Grape and grape products contain an array of polyphenolic compounds, and grape seeds are a concentrated source of polyphenolic flavan-3-ols existing in varying concentrations of monomeric (catechin (CT) and epicatechin (EC)),

Abbreviations: BP, blood pressure; CT, catechin; DBP, diastolic BP; EC, epicatechin; FMD, flow-mediated dilation; GSE, grape seed extract;

HOMA-IR, homoeostasis model assessment for insulin resistance; SBP, systolic BP.

* Corresponding author: B. Burton-Freeman, fax +1 3125675321 , email bburton@iit.edu 
dimeric, trimeric and polymeric tannin structures, depending on variety, processing, form, storage conditions and time ${ }^{(13)}$. Clinical trials supplementing with grape seed extracts (GSE) have shown clinically relevant reductions in BP in the order of $4 \cdot 1-8.7 \%$ for SBP and 3.4-9.0\% for $\mathrm{DBP}^{(14-17)}$. In vitro and ex vivo data suggest that GSE improves or enhances endothelial function through NO-mediated pathways ${ }^{(18)}$.

GSE is currently available as a capsule at dietary supplement stores. GSE in a food/beverage format has not been tested, largely due to poor solubility and unfavourable organoleptic properties. However, functional foods are a growing market for health-conscious consumers, driving advances in technology to overcome these challenges. The present study tested a patented GSE product in a beverage to lower BP in individuals with pre-hypertension. The primary end point was the difference in the change in SBP after 6 weeks of GSE beverage supplementation compared with a Placebo beverage. Secondary end points aimed to assess changes in DBP, markers of endothelial function, insulin sensitivity, markers of oxidative stress and inflammation, as well as to characterise GSE plasma phenolic components relative to efficacy outcomes.

\section{Methods \\ Study design}

The presented clinical trial was a single-centre, randomised, double-blinded, placebo-controlled, parallel study performed at the Clinical Nutrition Research Center at the Illinois Institute of Technology (Chicago, IL, USA). The study was conducted according to the guidelines laid down in the Declaration of Helsinki and the International Conference on HarmonizationGood Clinical Practice, and all the procedures involving human subjects were approved by the Institutional Review Board at the Illinois Institute of Technology. Written informed consent was obtained from all subjects before the initiation of the study. This trial was registered at clinicaltrials.gov as NCT00979732. The study was conducted including analyses from 2010 to 2014 .

\section{Subjects}

Eligible subjects were men and women with pre-hypertension (SBP between 120 and $139 \mathrm{mmHg}$ or DBP between 80 and $89 \mathrm{mmHg}$ ) between the ages of 25 and 65 years who had no clinical evidence or documented history of CVD, respiratory, renal, gastrointestinal or hepatic disease. Subjects who reported unusual dietary habits (e.g. pica), were actively losing weight or trying to lose weight, addicted to drugs or alcohol or smoked, presented with significant psychiatric or neurological disturbances or who were taking over-the-counter antioxidant supplements or prescription medications that may interfere with study procedures or end points were not eligible for participation. Past smokers were allowed to participate in the study if cessation occurred more than 2 years ago. Each subject was studied once. A total of thirty-six subjects (nineteen men and seventeen women) were enrolled in the study; twenty-nine (fifteen men and fourteen women) completed all the protocol-specified procedures (Fig. 1).

\section{Beverage treatments}

A purified GSE (MegaNatural ${ }^{\circledR}$ BP; Polyphenolics) with Generally Recognized as Safe (GRAS) status (FDA (GRAS Notice no. GRN 000125, dated 18 August 2003, FDA, USA)) was formulated in a fruit-based blend of apple, red grape, pomegranate and raspberry juices. The volume of the beverages was $12 \mathrm{fl} \mathrm{oz}$. $(355.0 \mathrm{ml})$ and contained $167 \mathrm{~kJ}$ (40 kcal). Beverages contained $0 \mathrm{~g}$ GSE (placebo) or $150 \mathrm{mg}$ GSE with identical appearance and similar taste. The juice base contained $333 \mu \mathrm{g} / \mathrm{ml}$ total polyphenols, $0.5 \mathrm{mg} / \mathrm{l}$ gallic acid and non-detectable amounts of CT and EC (mg/l). Adding the GSE (150 mg) to the base beverage resulted in $744 \mu \mathrm{g} / \mathrm{ml}$ total polyphenols, $14 \mathrm{mg} / \mathrm{l}$ gallic acid, $18 \mathrm{mg} / \mathrm{l} \mathrm{CT}$ and $14 \mathrm{mg} / \mathrm{l} \mathrm{EC}$. Total brix, acid and $\mathrm{pH}$ for the Placebo/control beverage and GSE-containing beverage was as follows: brix, 3.11 and 3.18; acidity, 5.92 and 5.88 g/l; and $\mathrm{pH}$, $2 \cdot 91$ and $2 \cdot 94$, respectively (data provided by Polyphenolics Inc. $\left.{ }^{(19,20)}\right)$. Subjects drank the beverages twice per day for a total daily dose of $0 \mathrm{mg} / \mathrm{d}$ GSE (Placebo) or $300 \mathrm{mg} / \mathrm{d}$ GSE. Beverages were provided by Coca-Cola Company, on behalf of Polyphenolics. The use of $300 \mathrm{mg} / \mathrm{d}$ dose is based on our previous finding that inclusion of the same dose significantly reduced $\mathrm{BP}$ in the metabolic syndrome patients and pre-hypertension individuals ${ }^{(14,17)}$. Beverages were prepared in three batches on three different occasions based on the dispensing plan of randomisation over the study period and stored in refrigerators with limited light exposure. The products were provided by the manufacturer with a unique randomisation code. Coded allocation envelopes for double-blinded randomisation were opened sequentially to assign subjects to respective treatments. Identity of the codes (Placebo or GSE) were kept in a sealed envelope until all the analyses were completed.

\section{Study timeline}

Eligible subjects started with a 2-week run-in period drinking the Placebo beverage twice per day. After the 2-week run-in period, subjects were randomised to one of the two groups (Placebo or GSE beverage), equally allocated to each treatment (1:1 randomisation ratio), according to a computer-generated randomisation scheduled by the study statistician. Subjects were counselled to consume their assigned beverages once in the morning and once in the evening preferably with meals. Subjects returned to the clinic biweekly to return all used and unused beverage bottles and to pick up their next 2-week supply. Compliance was documented by counting beverages consumed, and health status was assessed by documenting any adverse events. The follow-up phase was for 4 weeks with no treatment. Adequate allocation concealment remained until the study was concluded. Subjects were asked to maintain their usual level of physical activity and dietary habits throughout the study period. Dietary records were maintained by the subjects periodically throughout the study and reviewed by a staff dietitian to ensure compliance with usual dietary patterns. 


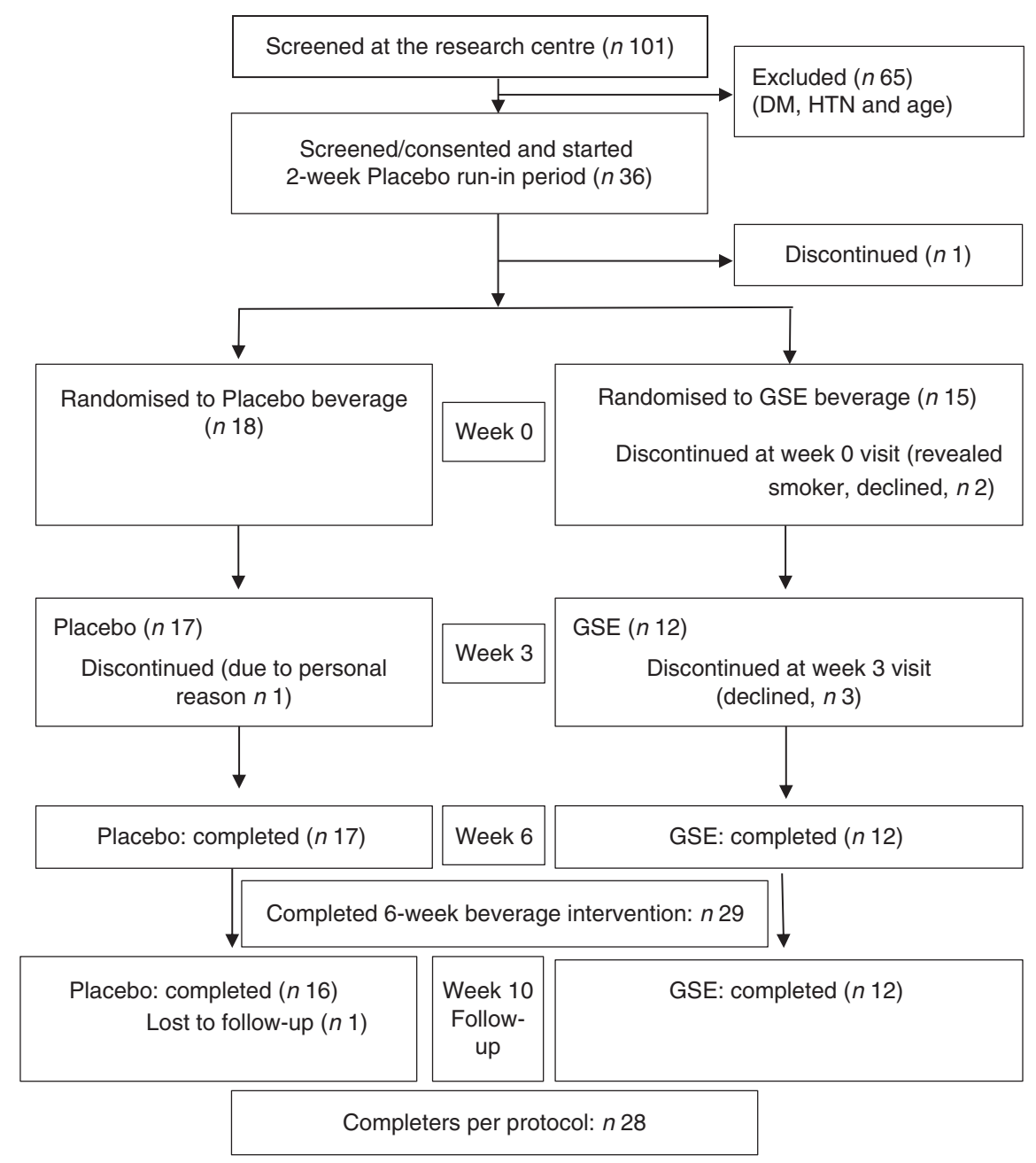

Fig. 1. Study progress: flow diagram of the participants in the 12-week grape seed extract (GSE) study. DM, diabetes mellitus; HTN, hypertension.

\section{Blood pressure measurement}

BP was monitored using ambulatory BP monitors (Ambulo 2400; Tiba Medical, Inc.) $)^{(21)}$ that were programmed to take BP and heart rate measurements automatically at 1-h intervals for a period of $24 \mathrm{~h}$. BP measurements were scheduled at the screening visit (to determine eligibility) and at week 0, 6 and 10 of the intervention (for efficacy assessment). Day-time BP and night-time BP were divided based on the subject's sleeping hours and hours awake, as indicated in their diaries, and were reviewed by the staff. Data were reviewed blinded, and when more than one measurement was recorded per hour the closest evaluable value to the hourly schedule was considered.

\section{Metabolic responses}

After 10-12 h of overnight fasting, fasting blood samples were collected at week 0 (baseline) and week 3, 6 and 10 of the intervention. Several analytical methods were used to assess the effects of GSE beverage consumption on plasma lipids, glucose, insulin, oxidised LDL (Ox-LDL) and soluble intercellular adhesion molecule-1 (sICAM-1). Plasma lipids and glucose concentrations were measured using standardised enzymebased assay kits (Randox) using the Randox Daytona auto clinical analyser (Randox). Plasma insulin concentrations were measured using the AlphaLISA method (PerkinElmer). Plasma Ox-LDL concentrations were measured using ELISA assay kits (Mercodia Inc.). Plasma sICAM-1 concentration was measured by highly sensitive ELISA assays (R\&D Systems). All assay protocols were performed according to the manufacturers' instructions and appropriate quality controls were used as applicable. Homoeostasis model assessment for insulin resistance (HOMA-IR) was calculated using fasting insulin $(\mu \mathrm{U} / \mathrm{l})$ and fasting glucose $(\mathrm{mmol} / \mathrm{l})$ concentration divided by $22 \cdot 5^{(22)}$.

\section{Flow-mediated dilation}

Flow-mediated vascular reactivity was studied in the brachial artery using methods recommended by the American College of Cardiology ${ }^{(23)}$. In brief, in a fasted state, the brachial artery was imaged by ultrasound (GE LOGIQ; GE Healthcare) and baseline measurements of vessel diameter $(\mathrm{mm})$ were collected. 
A BP cuff was then applied below the elbow for cuff inflation to a pressure of $50 \mathrm{mmHg}$ above SBP for $5 \mathrm{~min}$. Immediately after cuff deflation, brachial artery vessel diameter was monitored and measured to obtain peak vessel relaxation. Ultrasound measurements were recorded as six individual measurements of vessel diameter. All measurements took place in the morning after an overnight fast and in a dim-lit, temperature-controlled room. Subjects refrained from intense physical activity and consuming caffeinated drinks, tea, coffee and wine for $24 \mathrm{~h}$ before their laboratory visit. Flow-mediated dilation (FMD) measurements were scheduled at week 0 and 6 .

\section{Height and weight}

Height was measured to the nearest $0.5 \mathrm{~cm}$ using a wallmounted stadiometer (Ayrton Corp.) and weight (light clothing only) was measured to the nearest $0 \cdot 1 \mathrm{~kg}$ using digital scales. These data were used to derive BMI $\left(\mathrm{kg} / \mathrm{m}^{2}\right)$.

\section{Analysis of plasma phenolic compound and metabolites}

Chemicals. 5-(4-Hydroxyphenyl) pentanoic acid (4-hydroxyphenylvaleric acid) was obtained from Alfa Aesar, 3-(3-hydroxyphenyl) propionic acid was purchased from Lancaster Synthesis Inc. and 3,4-dihydroxyphenylacetic acid, 3-hydroxyphenylacetic acid and 4-hydroxybenzoic acid were obtained from Acros Organics. Ferulic acid was obtained from Calbiochem, and hydroxyphenylpropionic acid, 3,4-dihydroxyphenylpropionic acid, 3-methoxy-4-hydroxyphenylacetic acid (homovanillic), 4-hydroxyphenylacetic acid, $m$-coumaric acid, p-coumaric acid, caffeic acid, gallic acid, benzoic acid, hippuric acid, methyl hippuric acid, protocatechuic acid, vanillic acid, 3-hydroxybenzoic acid, 4-hydroxyhippuric acid, 3-hydroxyhippuric acid, 3,6-dimethoxybenzoic acid, (-)-EC, $(+)$-CT and taxifolin were purchased from Sigma-Aldrich. 5-Phenylvaleric acid and syringic acid were obtained from the University of East Anglia (C Kay, personal communication). Water was prepared using a Milli Q system (EMD Millipore) to $18 \mathrm{M} \Omega$ resistance. All the solvents used for sample extraction and chromatography analysis as well as $0 \cdot 22-\mu \mathrm{m}$ syringe filters were obtained from Fisher Scientific.

\section{Extraction of phenolic metabolites from plasma samples.}

Plasma samples were extracted using solid-phase extraction (SPE; Oasis HLB; Waters Corp.). In brief, the SPE cartridges were conditioned with $1 \mathrm{ml}$ of $\mathrm{MeOH}$ followed by $1 \mathrm{ml}$ of $1 \%$ (v/v) formic acid. Taxifolin and 3, 5-dimethylbenzoic acid $(10 \mu \mathrm{g} / \mathrm{ml})$ as internal standards were spiked in $1 \mathrm{ml}$ of plasma sample and were loaded into cartridges. The cartridges were then washed with $1 \mathrm{ml}$ of $1 \%(\mathrm{v} / \mathrm{v})$ formic acid. The phenolic acids were eluted with $1 \mathrm{ml}$ of $\mathrm{MeOH}$. The eluate was evaporated to dryness using Eppendorf Vacufuge ${ }^{\mathrm{TM}}$ (Eppendorf North America) and residues were reconstituted with $100 \mu \mathrm{l}$ of $0 \cdot 1 \%$ $(\mathrm{v} / \mathrm{v})$ formic acid in water.

Analysis of phenolic metabolites. The analysis of phenolic acid metabolites was carried out on a PerkinElmer Flexar HPLC.
The analytical column used was a Phenomenex Kinetex PFP Column $(4.6 \times 100 \mathrm{~mm}, 2 \cdot 6-\mu \mathrm{m}$ particle size $)$, operating at $40^{\circ} \mathrm{C}$ at a flow rate of $0.3 \mathrm{ml} / \mathrm{min}$, and the mobile phases consisted of (A) water-formic acid (99.9:0·1, v/v) and (B) acetonitrile-formic acid (99.9:0.1, v/v). The separation was performed using the gradient elution programme: $0 \mathrm{~min}, 2 \% \mathrm{~B}$; $12 \mathrm{~min}, 20 \% \mathrm{~B}$; $17 \mathrm{~min}, 50 \% \mathrm{~B}$; and held for $3 \mathrm{~min}$ at $100 \% \mathrm{~B}$, followed by 5 min of re-equilibration of the column before the next run. The injection volume was $10 \mu$ l. The HPLC system was coupled with an $\operatorname{AxION}^{\circledR} 2$ ToF Mass Spectrometer (PerkinElmer) equipped with an ESI interface operating in negative ion trap pulse mode. The MS parameters were as follows: flight tube: $10 \mathrm{kV}$; trap pulse mode: 100-450 m/z; IG exit low (D7): $18 \mu \mathrm{s}$, trap pulse delay (D8): $28 \mu \mathrm{s}$; capillary exit voltage: $-80 \mathrm{~V}$; drying gas heater: $350^{\circ} \mathrm{C}$; drying gas flow: 15 litres/min; and nebulising gas pressure: 80 PSI. Liquid $\mathrm{N}_{2}$ was used as drying gas. Standard curves were prepared at six different concentrations $(0 \cdot 05-20 \mu \mathrm{g} / \mathrm{ml})$ for quantitation of phenolics in plasma.

\section{Statistical analysis}

Subject characteristics were analysed using descriptive statistics from data collected at the screening visit. Group comparisons of continuous variables were carried out using independent (unpaired) Student's $t$ tests and categorical variables using a $\chi^{2}$ test. Results were presented as numbers and percentages, as appropriate. Mixed model analysis of repeated measures was performed on each quantitative outcome variable to test main effects of beverage treatment (GSE, Placebo) and time (weeks) using PROC MIXED via Window PC-SAS (version 9.3; SAS Institute Inc.). For testing and selecting best covariance structure, model comparisons were examined using -2 loglikelihood scores and Akaike information criterion values from each structure. In the final analyses, treatment, time and interaction of treatment and time were included and the corresponding baseline value was included as a covariate. The Kenward-Roger correction and the method of restricted maximum likelihood were used in all mixed models. Independent sample two-tailed $t$ tests were used to compare the magnitudes of change $(\Delta)$ from week 0 to week 6 and from week 6 to week 10 in SBP and DBP. Continuous variables were examined for normality, and data not conforming to normal distribution patterns were log-transformed before analysis. Outlier removal may have resulted in fewer evaluable subjects for secondary end points and were indicated accordingly. In a post hoc analysis of BP responses, median values of SBP and DBP at week 0 were used to split the subjects into groups with higher than or equal to the median value $v$. lower than median value and analyses were performed as noted above (mixed model and independent samples $t$ tests).

Non-parametric Mann-Whitney test was performed to compare non-normally distributed total plasma concentration of phenolic compounds (GSE $v$. Placebo) at week 0, 6 and 10 using SPSS (version 19.0; IBM). Correlational analysis was conducted between total phenolic metabolites and changes in BP and metabolic outcome measurements using Spearman's correlation in SAS. The results of the statistical analyses are presented as least squares means (LSM) with their standard 
Table 1. Baseline characteristics of the study participants* (Mean values and standard deviations; numbers and percentages)

\begin{tabular}{|c|c|c|c|c|}
\hline \multirow[b]{3}{*}{ Variables } & \multicolumn{4}{|c|}{ Total $(n$ 29) } \\
\hline & \multicolumn{2}{|c|}{ Placebo beverage $(n 17)$} & \multicolumn{2}{|c|}{ GSE beverage ( $n$ 12) } \\
\hline & Mean & SD & Mean & SD \\
\hline Age (years) & 42 & 10 & 44 & 10 \\
\hline Height $(\mathrm{cm})$ & 170 & 10 & 172 & 7 \\
\hline Weight (kg) & 89 & 25 & 101 & 19 \\
\hline $\mathrm{BMI}\left(\mathrm{kg} / \mathrm{m}^{2}\right)$ & 31 & 9 & 34 & 7 \\
\hline Fasting glucose concentration $(\mathrm{mmol} / \mathrm{l})$ & 4.83 & 0.50 & 5.16 & 0.67 \\
\hline Clinical systolic blood pressure (mmHg) & 128 & 9 & 128 & 7 \\
\hline Clinical diastolic blood pressure $(\mathrm{mmHg})$ & 84 & 8 & 82 & 6 \\
\hline \multirow[t]{2}{*}{ Umbilicus waist circumference $(\mathrm{cm})$} & 101 & 19 & 109 & 22 \\
\hline & $n$ & $\%$ & $n$ & $\%$ \\
\hline \multicolumn{5}{|l|}{ Ethnicity } \\
\hline Caucasian & 6 & 35 & 4 & 33 \\
\hline African-American & 7 & 41 & 7 & 58 \\
\hline Asian & 2 & 12 & 1 & 8 \\
\hline Hispanic & 2 & 12 & 0 & 0 \\
\hline \multicolumn{5}{|l|}{ Sex } \\
\hline Women & 8 & 47 & 6 & 50 \\
\hline Men & 9 & 53 & 6 & 50 \\
\hline
\end{tabular}

GSE, grape seed extract.

${ }^{*}$ Independent (unpaired) samples $t$ tests and $x^{2}$ test showed no significant differences in baseline measures between groups.

errors unless indicated otherwise. Statistical significance was based on two-sided treatment comparison at the $5 \%$ significance level under a null hypothesis of no difference between treatments.

Sample size estimates were based on the primary end point of SBP and the following assumptions: two treatments, mean difference in SBP between GSE and Placebo of $8 \mathrm{mmHg}$ after 6 weeks of treatment and standard deviation of 8 . Power calculations indicated a total sample size of thirty-four subjects (randomised in a 1:1 ratio to GSE or Placebo), assuming that $20 \%$ attrition would provide $>80 \%$ power with a level of 0.05 to detect significant differences in SBP between GSE and Placebo beverages.

\section{Results}

\section{Demographic and baseline characteristics}

A total of thirty-six subjects were recruited into the study; twenty-nine subjects completed 6 weeks of GSE or Placebo ( $n 12$ and $n$ 17, respectively) and twenty-eight subjects completed the entire study, including the 4 -week no beverage follow-up period (one subject from the Placebo group was lost to follow-up) (Fig. 1). The present study was conducted between July 2010 and June 2012.

Baseline characteristics of study subjects completing 6 weeks of GSE or Placebo ( $n$ 29) are presented in Table 1. Groups were well balanced; no statistical differences were observed between GSE and Placebo groups for age, BP, BMI, waist circumference, race/ethnicity or sex (GSE $v$. Placebo comparisons at baseline: all $P>0.05$ ). Body weights in both groups were maintained consistently throughout the study period.
Subjects' dairy and returned bottle counts indicated that compliance of beverage consumption was between 80 and $100 \%$ in both groups. Drinks were well liked and tolerated. GSE was found to be safe and no adverse events related to treatments were reported during the study.

\section{Blood pressure}

BP measurements were recorded for $24 \mathrm{~h}$ and split between day/ awake-time and night/sleep-time for analysis (see 'Methods' section).

Day/awake-time blood pressure. SBP and DBP were not different between the GSE and Placebo groups at the screening or at baseline visits (week $0 ; P>0.05$ ). BP did not significantly change in either group from screening to week 0 visits $(P>0.05)$ or after the 2-week run-in with Placebo beverage.

Systolic blood pressure $(\mathrm{mmHg})$. Consumption of the GSE beverage for 6 weeks led to a significant reduction in SBP (week 0: 125.0 (SEM 2.3) $v$. week 6: 118.0 (SEM 2.3) $\mathrm{mmHg}$; $P=0.02$, Table 2 ), whereas consumption of the Placebo beverage resulted in no change in SBP after 6 weeks $(P>0.05$, Table 2). The mean SBP of GSE- and Placebo-treated groups at week 6 was significantly different (118.0 (SEM 2.3) v. 127.0 (sem 1.9$) \mathrm{mmHg}$, respectively; $P=0.003$, Table 2 ). The magnitude of reduction in SBP after consumption of GSE $v$. Placebo beverage for 6 weeks was -7 (SEM 3.2) v. 1.4 (SEM 2.1) $\mathrm{mmHg}$, respectively $(P=0 \cdot 03)$. After cessation of treatment beverages, mean SBP at week 10 increased in the GSE group to levels $(\mathrm{mmHg})$ not different from baseline (125.0 (SEM 2.3) at week $0 v$. $124 \cdot 1$ (SEM 2.0) $\mathrm{mmHg}$ at week 10 , respectively $(P>0 \cdot 05)$ ). 
Table 2. Effect of beverage consumption on day-time and night-time blood pressure (Least squares means (LSM) with their standard errors)

\begin{tabular}{|c|c|c|c|c|c|c|}
\hline \multirow[b]{2}{*}{ Blood pressure } & \multirow[b]{2}{*}{ Week } & \multicolumn{2}{|c|}{ Placebo } & \multicolumn{2}{|c|}{ GSE } & \multirow[b]{2}{*}{$P^{*}$} \\
\hline & & LSM & SEM & LSM & SEM & \\
\hline \multicolumn{7}{|l|}{ Day-time $\dagger$ variable } \\
\hline \multirow[t]{3}{*}{ Systolic blood pressure $(\mathrm{mmHg})$} & 0 & $125 \cdot 6^{\mathrm{a}}$ & 1.9 & $125 \cdot 0^{\mathrm{a}}$ & $2 \cdot 3$ & 0.83 \\
\hline & 6 & $127 \cdot 0^{\mathrm{a}}$ & 1.9 & $118 \cdot 0^{\mathrm{b}}$ & $2 \cdot 3$ & 0.003 \\
\hline & 10 & $123 \cdot 7^{\mathrm{a}}$ & $2 \cdot 0$ & $124 \cdot 1^{\mathrm{a}}$ & $2 \cdot 0$ & 0.88 \\
\hline \multirow[t]{3}{*}{ Diastolic blood pressure (mmHg) } & 0 & $82 \cdot 9$ & $1 \cdot 3$ & $83 \cdot 0$ & 1.6 & 0.99 \\
\hline & 6 & $83 \cdot 6$ & $1 \cdot 3$ & $79 \cdot 1$ & 1.6 & 0.08 \\
\hline & 10 & $81 \cdot 3$ & 1.4 & 81.9 & 1.6 & 0.78 \\
\hline \multicolumn{7}{|l|}{ Night-time $†$ variable } \\
\hline \multirow[t]{3}{*}{ Systolic blood pressure $(\mathrm{mmHg})$} & 0 & $113 \cdot 1$ & $2 \cdot 0$ & $112 \cdot 9$ & $2 \cdot 3$ & 0.94 \\
\hline & 6 & 111.2 & 2.0 & 111.5 & 2.5 & 0.94 \\
\hline & 10 & 114.7 & $2 \cdot 1$ & $114 \cdot 3$ & 2.4 & 0.91 \\
\hline \multirow[t]{3}{*}{ Diastolic blood pressure $(\mathrm{mmHg})$} & 0 & $73 \cdot 1$ & 1.5 & $73 \cdot 1$ & 1.7 & 0.98 \\
\hline & 6 & $74 \cdot 7$ & 1.7 & $72 \cdot 6$ & 1.9 & 0.40 \\
\hline & 10 & $74 \cdot 8$ & 1.5 & $72 \cdot 1$ & 1.8 & 0.26 \\
\hline
\end{tabular}

GSE, grape seed extract.

a,b Mean values with unlike superscript letters were significantly different $(P<0.05)$

* $P$-value represents between-treatment comparison of Placebo $v$. GSE at each week based on the mixed model.

† Day-time results: GSE - week 0, 6, 10, $n$ 12; Placebo - week 0, 6, $n 17$; week 10, $n$ 16; night-time results: GSE - week $0, n 11$; week $6, n$ 9; week 10, $n$ 10; Placebo - week 0 , $n$ 15; week $6, n 13$; week $10, n 14$.

Table 3. Effect of beverage consumption on day-time blood pressure in the median blood pressure groups (Least squares means (LSM) with their standard errors)

\begin{tabular}{|c|c|c|c|c|c|c|}
\hline \multirow[b]{2}{*}{ Variables } & \multirow[b]{2}{*}{ Week } & \multicolumn{2}{|c|}{ Placebo* } & \multicolumn{2}{|c|}{ GSE* $^{*}$} & \multirow[b]{2}{*}{$P+$} \\
\hline & & LSM & SEM & LSM & SEM & \\
\hline \multicolumn{7}{|l|}{ SBP (median of $125.1 \mathrm{mmHg}$ ) } \\
\hline \multirow[t]{2}{*}{ Above the median ( $n$ 14) } & 0 & $132 \cdot 3^{a}$ & $2 \cdot 1$ & $132 \cdot 4^{\mathrm{a}}$ & 2.4 & 0.97 \\
\hline & 6 & $132 \cdot 3^{a}$ & $2 \cdot 1$ & $118 \cdot 9^{\mathrm{b}}$ & $2 \cdot 4$ & 0.0003 \\
\hline \multirow[t]{2}{*}{ Below the median ( $n 15)$} & 0 & $119 \cdot 0$ & $2 \cdot 3$ & $118 \cdot 6$ & $2 \cdot 9$ & 0.93 \\
\hline & 6 & 121.5 & $2 \cdot 3$ & $118 \cdot 2$ & 2.9 & 0.40 \\
\hline \multicolumn{7}{|l|}{ DBP (median of $83.5 \mathrm{mmHg}$ ) } \\
\hline \multirow[t]{2}{*}{ Above the median ( $n$ 14) } & 0 & $88 \cdot 2^{\mathrm{a}}$ & $1 \cdot 3$ & $88 \cdot 7^{\mathrm{a}}$ & $1 \cdot 8$ & 0.82 \\
\hline & 6 & $89 \cdot 2^{a}$ & $1 \cdot 3$ & $83 \cdot 0^{\mathrm{b}}$ & $1 \cdot 8$ & 0.01 \\
\hline \multirow[t]{2}{*}{ Below the median ( $n 15)$} & 0 & $77 \cdot 7$ & 1.9 & $78 \cdot 0$ & $2 \cdot 0$ & 0.93 \\
\hline & 6 & $78 \cdot 1$ & 1.9 & 75.5 & $2 \cdot 0$ & 0.37 \\
\hline
\end{tabular}

GSE, grape seed extract; SBP, systolic blood pressure; DBP, diastolic blood pressure; SEM, standard errors of the mean in subjects qualifying above or below the group median for corresponding blood pressure $(\mathrm{mmHg})$.

a,b Mean values with unlike superscript letters were significantly different $(P<0.05)$.

* Above the median SBP (GSE, $n$ 6; Placebo, $n$ 8); below the median SBP (GSE, $n$ 6; Placebo, $n$ 9). Above the median DBP (GSE, $n$; Placebo, $n$ 9); below the median DBP (GSE, $n$ 7; Placebo, $n$ 8).

† $P$-value represents between-treatment comparison of Placebo $v$. GSE at each week, based on the mixed model.

No significant changes were observed in SBP in subjects assigned to the Placebo beverage.

Diastolic blood pressure ( $\mathrm{mmHg}$ ). Consumption of GSE beverage for 6 weeks lowered the mean DBP from 83.0 (SEM 1.6) to $79 \cdot 1$ (SEM 1.6) $\mathrm{mmHg}$; however, this was not statistically significant (Table 2 ). The magnitude of reduction in DBP after consumption of GSE beverage for 6 weeks was -3.8 (SEM 1.5) v. 0.7 (SEM 1.8) $\mathrm{mmHg}$ after the Placebo beverage $(P=0.08)$. No significant differences between groups in DBP were observed at week $10(P>0.05)$.

In the subgroup analyses, subjects were categorically defined above or below the groups' median BP values based on mean baseline (week 0) values (Table 3). The SBP median was $125 \mathrm{mmHg}$, and the DBP median was $83.5 \mathrm{mmHg}$.

Below the systolic blood pressure and diastolic blood pressure medians. There was no significant effect of consumption of the
GSE or Placebo beverage for 6 weeks on SBP or DBP in the subgroup that fell below the group median $(P>0.05)$.

Above the systolic blood pressure and diastolic blood pressure group medians $(\mathrm{mmHg})$. The mean treatment and treatmentby-week interaction for SPB was $P=0.007$ and 0.006 , respectively. There was a significant effect of consumption of the GSE beverage for 6 weeks on SBP and DBP $(P=0.0006$ and 0.03 , respectively), whereas no effect was observed with the Placebo beverage. The magnitude of SBP change from week 0 to week 6 was -13.6 (SEM 3.9) $\mathrm{mmHg}$ for the GSE group and 0.01 (SEM $2 \cdot 8$ ) $\mathrm{mmHg}$ for the Placebo group $(P=0 \cdot 01)$.

The mean treatment and treatment-by-week interaction for DBP was $P=0.007$ and 0.006 , respectively. The magnitude of DBP change from week 0 was -5.8 ( $\mathrm{sEM} 2.0$ ) $\mathrm{mmHg}$ for the GSE group and 1.0 (SEM $2 \cdot 2$ ) $\mathrm{mmHg}$ for the Placebo group $(P=0 \cdot 06)$. 
Night/sleep-time blood pressure. Data available for assessment of night/sleep-time BP were limited, averaging 5 (SEM 2) (range 0-9) evaluable measurements during sleep due to error in readings, multiple measurement attempts and subject removal of cuff (complaints of sleep disturbance). Accordingly, variance and standard deviation was increased during night/sleep measurements. No significant differences between treatments at week 0,6 or 10 were observed (Table 2; $P>0.05)$.

\section{Flow-mediated dilation}

The mean percentage change in FMD from week 0 to week 6 after the GSE beverage was 7.4 (SEM 1.5) v. 7.7 (SEM 1.5) \%, respectively $(P>0.05)$. The mean percent change after the Placebo intervention was 9.9 (SEM 1.3) v. 8.2 (SEM 1.3 ) \% $(P>0 \cdot 05)$. No significant differences were apparent between treatments on FMD at week 6 (data not shown).

\section{Biochemical markers}

No significant effects of treatments on mean plasma concentrations of glucose, lipids, Ox-LDL or SICAM-1 were evident (Table 4). Fasting plasma insulin concentration and HOMA-IR at baseline indicated that most of subjects were hyperinsulinaemic or insulin resistant. No differences in glucose concentrations were evident (Fig. 2); however, fasting plasma insulin concentrations tended to be lower at week 6 after consuming the GSE beverage $v$. the Placebo beverage ( $P=0 \cdot 09$, Fig. 3 ), and improvements in insulin sensitivity as calculated by HOMA-IR were apparent $(P=0 \cdot 07$, Fig. 4).

\section{Phenolic metabolites}

Fig. 5 shows the mean changes in total phenolic acid metabolite concentrations in plasma after Placebo and GSE beverages from week 0 to 6 and then week 10. The mean total plasma phenolic metabolite concentrations at week 0 was $5819 \cdot 8$ (sD 325.4) $\mu \mathrm{g} / 1$ and increased to $138317 \cdot 2$ (SD $37977 \cdot 2$ ) $\mu \mathrm{g} / \mathrm{l}$ after 6 week of GSE consumption, which was approximately 1.6 higher than the mean total phenolic acid concentrations after the Placebo beverage at week $6(87895.8$ (SD 20 531.4) $\mu \mathrm{g} / \mathrm{l}$ ). Among the individual metabolites, the largest fold change was observed with hippuric acid, which resulted in an approximately 40-fold change from week 0 to week 6 after the GSE beverage and 25-fold change after the Placebo beverage (Table 5). Other distinguishing metabolites marking GSE consumption were 3-hydroxyphenylacetic acid, 4-hydroxyhippuric acid and homovanillic acid, which showed relative increases in plasma after 6 week of GSE consumption compared with the Placebo beverage (Table 5). Despite measurable changes, the changes in individual metabolites and total phenolic acid metabolites were not significantly different between GSE and Placebo beverages at 0 or 6 week $(P>0.05)$. Likewise, no differences were observed between beverages at week 10, although a decline in total phenolic acid metabolites was observed after discontinuation of the beverages.

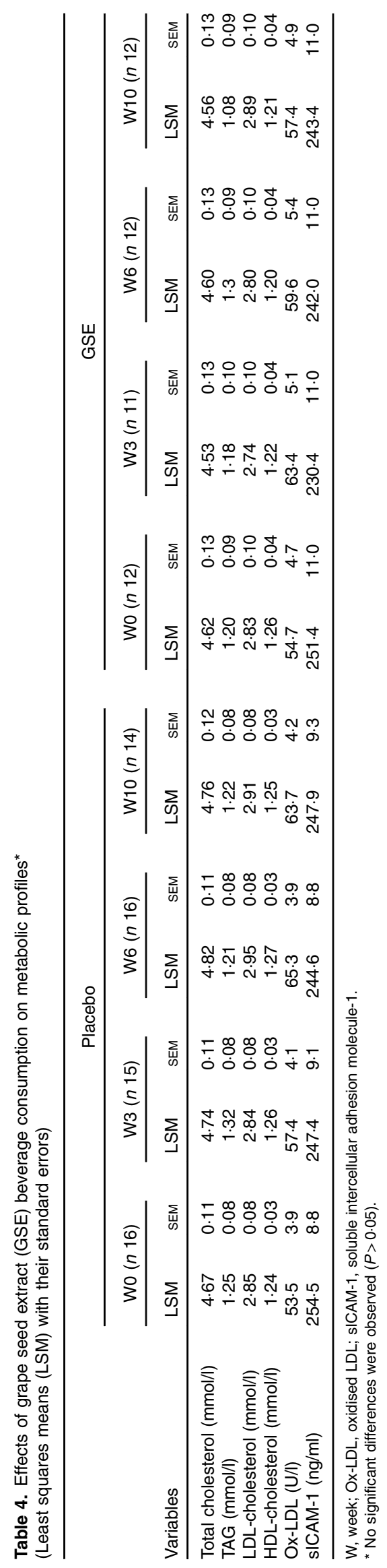




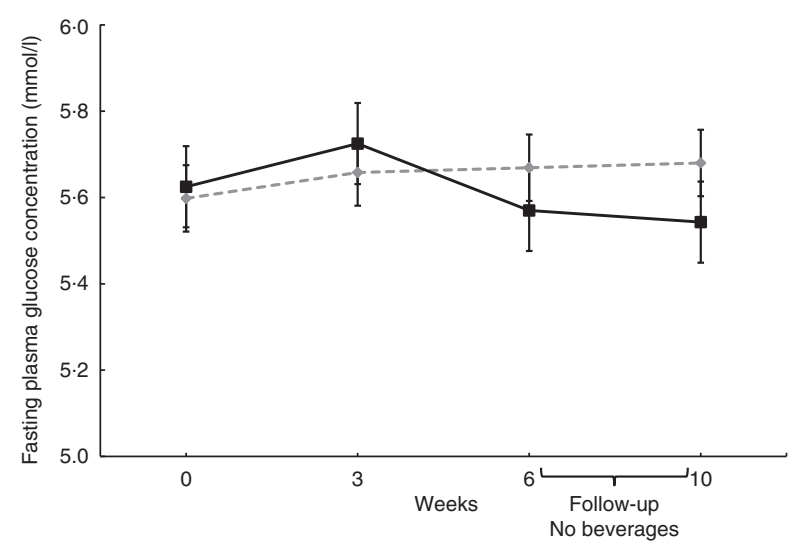

Fig. 2. Effect of the grape seed extract (GSE) beverage on fasting glucose concentrations over the 6-week and 4-week follow-up period. Mixed model adjusted for the corresponding baseline value showed no significant differences between groups (week 0 to week $6, n$ 29; 4-week follow-up, $n$ 28). Values are means (least squares means), with their standard errors. $-{ }_{-}-$, Placebo; —-, GSE.

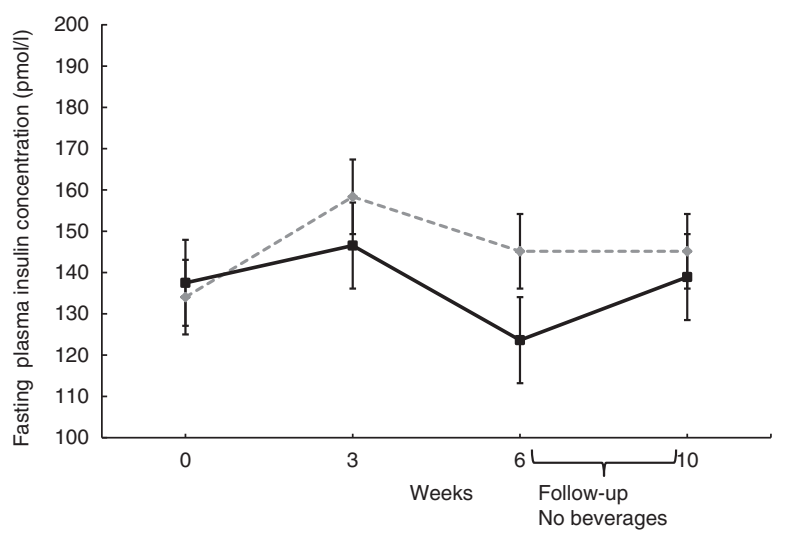

Fig. 3. Effect of grape seed extract (GSE) beverage on fasting insulin concentrations over the 6-week and 4-week follow-up. Mixed model was used and adjusted for the corresponding baseline value (week 0 to week 6 , $n$ 29; 4-week follow-up, $n$ 28). Values are means (least squares means), with their standard errors. $-\multimap-$, Placebo; $\longrightarrow$, GSE.

No correlations were observed between the metabolites and any of the clinical measurements (data not shown).

\section{Discussion}

Functional foods are increasingly popular among consumers to maintain health and reduce the need for pharmacological intervention. The present study aimed to test GSE as a functional beverage to lower $\mathrm{BP}$ in people with pre-hypertension. Consumption of GSE included in a beverage for 6 weeks resulted in a mean reduction of day-time SBP and DBP by 5.6 and $4.7 \%$, respectively, compared with no change after consuming the same fruit juice-based beverage without GSE. Moreover, the BP-lowering effects of GSE were more pronounced in subjects with higher initial BP, which nearly doubled the effect size. After discontinuation of the GSE

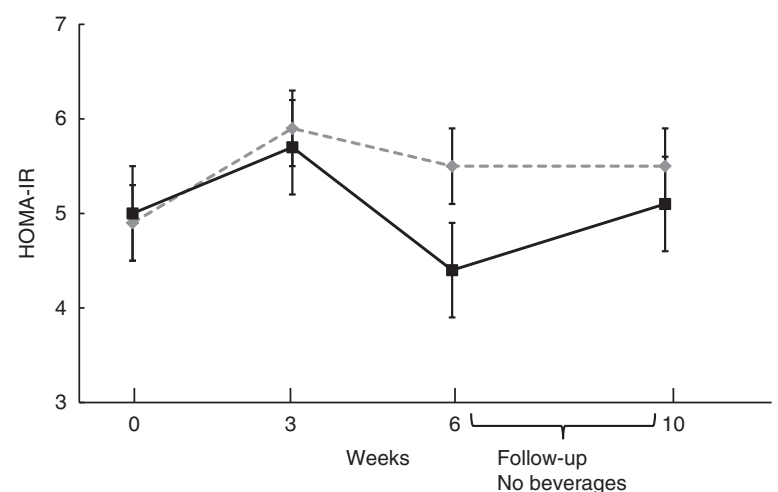

Fig. 4. Effect of grape seed extract (GSE) beverage on fasting homoeostasis model assessment for insulin resistance (HOMA-IR) over the 6-week and 4-week follow-up period. Mixed model was used and adjusted for the corresponding baseline value (week 0 to week $6, n$ 29; 4-week follow-up, $n$ 28). Values are means (least squares means), with their standard errors. -- , Placebo; — $\longrightarrow$ GSE.

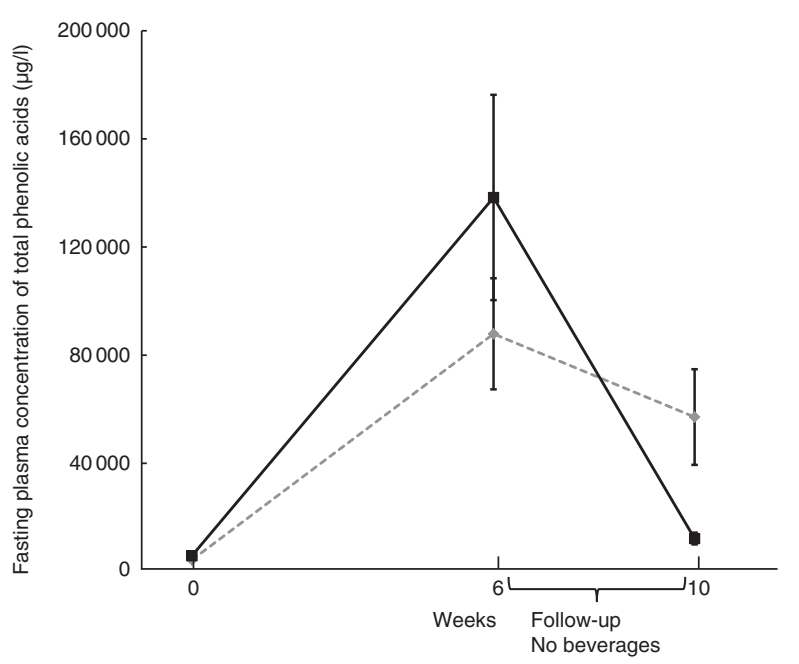

Fig. 5. Effect of grape seed extract (GSE) beverage on changes in plasma concentrations of total phenolic acids over the 6-week and 4-week follow-up period. Non-parametric Mann-Whitney test showed no differences between groups and over time within group (week 0 to week 6, n 29; 4-week follow-up, $n$ 28). Values are means and standard deviations. $-\downarrow--$, Placebo; $\longrightarrow-$, GSE.

beverage for 4 weeks, BP increased back to baseline pressures, verifying the biological activity of GSE on BP.

Several studies have shown that GSE improves $\mathrm{BP}^{(14,16,17,24)}$. However, not all studies with GSE have produced significant or beneficial changes in $\mathrm{BP}^{(15,25-27)}$. This may be due to compositional differences between GSE products ${ }^{(14,17,25)}$, populations being studied (i.e. older adults may be less responsive due to the aged endothelium) ${ }^{(15)}$, or dosing issues, including too high of a dose based on percentage of monomers ${ }^{(25,26)}$, combination with other therapies (e.g. diet and lifestyle management) ${ }^{(24)}$ or incompatible dose combinations such as vitamin $\mathrm{C}+\mathrm{GSE}^{(26)}$. Analysis of the plasma metabolites could also reveal important features related to efficacy. There are no data currently available characterising GSE phenolic metabolites in human plasma after 
E. Park et al.

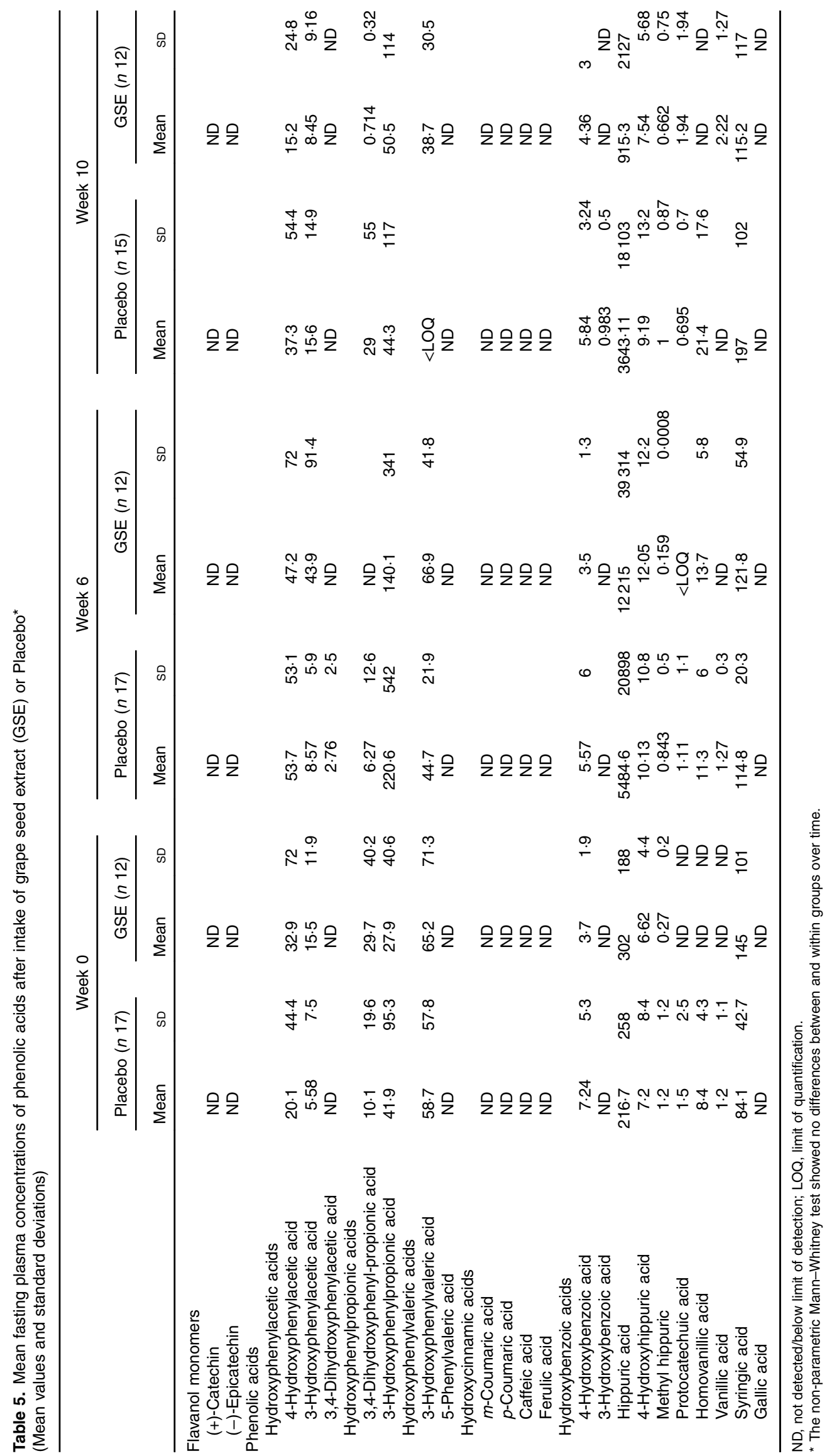


chronic consumption. In the current study, fasting samples were analysed. These samples represent late phase metabolites generated from the microbes of the lower bowel. Total phenolic metabolite concentrations were approximately 1.6 times higher in the GSE group at 6 weeks compared with the Placebo group and reduced back to baseline after beverage discontinuation an inverted pattern relative to mean changes in BP (i.e. decreased BP at 6 weeks and increased at 10 weeks). However, there was no significant correlation between the metabolites and BP response. The lack of significant correlation and differences between GSE and Placebo is likely due to the large inter-individual variability in plasma concentrations of all the phenolic acids, which have been demonstrated previously $^{(28,29)}$. Differences in gut microbiota composition related to dietary, environmental and host genetic variance are among the reasonable and plausible explanations for the observed responses ${ }^{(30,31)}$. The most abundant phenolic acids identified with GSE intake were hippuric acid, 3-hydroxyphenylacetic acid, 4-hydroxyhippuric acid and homovanillic acid; however, these phenolic acids may also be microbial end products from sources other than grape seed $^{(29,32)}$, explaining at least in part why some were also elevated in the Placebo beverage group, which had $333 \mu \mathrm{g} / \mathrm{ml}$ of total polyphenols from the fruit juice part of the beverage. Thus, although total and key phenolic metabolites increased with GSE supplementation, confirming compliance with drink consumption, there were no statistically definable metabolites to attribute the BP-lowering effects of GSE in this free-living population who were self-selecting their diets. Future investigations controlling all aspects of dietary intake (i.e. controlled feeding design) may reveal such information and unveil the role of dietary phenolic acids in cardiovascular health.

\section{Possible mechanisms of action}

MegaNatural ${ }^{\circledR}$ BP contains a mixture of monomers, oligomers and polymers of CT and EC. The composition ranges between 5.4 and $13.8 \%$ monomers (US Patent No. 8,075,929). The composition of GSE is very similar to $\mathrm{COCOa}^{(33)}$. Research with cocoa suggests that the monomeric flavanols EC and CT are responsible for the $\mathrm{BP}$ and vasoactive effects ${ }^{(34)}$. These monomeric flavanols peak in the plasma within the first several hours following consumption and are cleared by $12 \mathrm{~h}^{(35)}$. Similar to cocoa, the BP-lowering properties of GSE appear to be closely linked to the up-regulation of endothelial NO synthase (eNOS), resulting in increased endothelial $\mathrm{NO}$ and endothelialdependent vasodilation ${ }^{(18)}$. In humans, FMD is a functional marker of endothelial function by assessing endothelialdependent vasodilation. GSE consumption in the present study revealed no remarkable enhancing effect on FMD in the fasting state. The data are consistent with other research findings assessing effects of chronic GSE intake on vasodilation in the fasting state ${ }^{(26,36)}$. Several studies indicate that measuring enhanced vasodilation responses is best during peak concentrations of monomeric flavanols (CT and EC and their metabolites), which would occur $1-2 \mathrm{~h}$ after consumption $^{(34,37,38)}$. The present study measured FMD $12-14 \mathrm{~h}$ after the last dose of GSE or Placebo, during which monomeric flavanols were cleared and not detected in plasma (Table 5). It is possible that an enhanced FMD response would have been apparent if the procedure had been conducted during peak CT/EC concentrations. Accordingly, the data do not allow a direct conclusion that changes in endothelial relaxation responses were responsible for the BP-lowering effects of GSE observed in this study. However, in contrast to our data, Barona et al. ${ }^{(16)}$ observed significantly higher FMD responses $(P<0 \cdot 0001)$ along with significantly decreased SBP $(P<0.0025)$ in men with the metabolic syndrome after a $30-\mathrm{d}$ treatment with freeze-dried grape polyphenolic powder equivalent to two servings of grapes per $\mathrm{d}$. The structural differences of the compounds in GSE and freeze-dried grape polyphenolic powder may be responsible for the observed differences, although the product used in the present study delivered higher amounts of total phenolic compounds (approximately $528 v .126 \mathrm{mg} / \mathrm{d}$ ).

Other/additional mechanisms may be responsible for the effects of GSE on BP. These include inhibition of angiotensinconverting enzyme activity $^{(39)}$ or increasing endothelial NO bioavailability by inhibiting superoxide production from endothelial NADPH oxidase ${ }^{(40)}$. Vasorelaxation properties in response to GSE may also be achieved through insulinmediated signalling pathways ${ }^{(41)}$, which is of interest in the present study, given the observed trend in improvement in fasting insulin and insulin sensitivity calculated from the HOMA-IR. Evidence supports a link between hypertension and insulin resistance. The common mechanism, although not entirely understood and is often debated ${ }^{(42)}$, appears to rely on production and bioavailability of $\mathrm{NO}^{(43)}$. Under normal conditions, insulin interacts with its receptor and stimulates a phosphorylation cascade of proteins (e.g. insulin receptor substrate 1 (IRS-1), PI3/Akt kinase, eNOS) ultimately resulting in NO production causing relaxation of vascular smooth muscles. However, in the present study, data are not available to indicate increased $\mathrm{NO}$ or its by-products such as nitrate $\left(\mathrm{NO}^{3-}\right)$ and nitrite $\left(\mathrm{NO}^{2-}\right)$. The blood samples were collected after an overnight fast and $12-14 \mathrm{~h}$ since the last GSE beverage was consumed. Therefore, it is unlikely that significant changes in treatment-related markers of NO production would be observed in the fasting samples, unless microbial metabolites are effective in activating the eNOS signalling pathway, in which case significant changes in FMD might have been expected. Phenolic acids such as hippuric acid, hydroxyhippuric acid and homovanillic acid have been associated with FMD responses, but at markedly higher concentrations and not in the fasting state ${ }^{(44)}$.

Insulin also participates in Ca-mediated vasodilation, which includes effective glucose transport and phosphorylationenhancing $\mathrm{Ca}$ efflux and vasodilation. Alternatively, insulin resistance results in vascular resistance and elevated $\mathrm{BP}$ due to impaired PI3/Akt kinase activation and impaired Ca efflux ${ }^{(45,46)}$. Therefore, improving insulin sensitivity could presumably increase vasoreactivity and lower BP. Supportive epidemiology shows modest protective association between insulin sensitivity and risk of incident hypertension ${ }^{(47)}$ and higher risk of hypertension with higher fasting insulin concentrations in young women ${ }^{(48)}$. GSE has been shown to improve postprandial glucose responses in people with the metabolic 
syndrome ${ }^{(49)}$ as well as prevent insulin resistance in fructose-fed rats by improving cellular insulin signalling ${ }^{(50)}$. In a $15-\mathrm{d}$ intervention with flavanol-rich chocolate, Grassi et al. ${ }^{(51)}$ reported significantly decreased HOMA-IR, increased insulin sensitivity, increased FMD and decreased BP in men and women with essential hypertension and impaired glucose tolerance. In the present study, baseline insulin concentrations were elevated in most of the subjects ( $80 \%$ had baseline fasting insulin over the 75th percentile cut-off of $13.13 \mu \mathrm{U} / \mathrm{ml}$ ) and all subjects had baseline HOMA-IR values $>2 \cdot 5$. Study results showed significantly reduced $\mathrm{BP}$ in the GSE group compared with the Placebo group and a possible trend to have improved fasting insulin levels and HOMA-IR, implying that changes in BP may have been related to changes in insulin sensitivity.

\section{Strengths, limitations and future research}

Overall, in the present study, GSE was successfully formulated in a low-energy functional beverage that was easily incorporated into the diets of people with pre-hypertension, resulting in lower BP. These data provide a promising dietary behaviour approach to achieve and maintain healthy BP. Strengths of the study design included a run-in period and a follow-up period without active intervention to clarify GSE-specific effects. The study also included measurement of polyphenol contents in the plasma along with measurements of biomarkers associated with improved insulin resistance, oxidative stress, inflammation and endothelial function, which have been suggested as possible mechanisms for reduction of BP. A limitation of the study, however, was that, despite sufficient power to detect statistical differences on the primary end point of SBP, the study was not sufficiently powered to detect significant differences on the secondary end points. Therefore, it is possible at least partly that some of the observed trends in decreased secondary end points, specifically DBP and insulin resistance, did not meet statistical significance due to insufficient sample size. Similar issues were apparent for the plasma phenolic compounds. We observed large inter-individual variability in plasma phenolic concentrations, requiring a greater sample size to address variability issues. Further, the complete absorption and metabolic kinetics of GSE constituents are not fully understood. Future investigations are warranted to address questions specific to efficacy, bioavailability and complete metabolite profiles. Finally, a strength and limitation of the study was that we did not control the diets of subjects. Therefore, effects of the GSE beverage were observed in a real-world situation: free-living group of people with pre-hypertension. However, this may also be a reason for some of the variability seen in secondary end points. Future studies with dietcontrolled conditions or greater sample size would help address this point.

The present study supports the use of GSE as a functional ingredient in a low-energy beverage to reduce the risk of hypertension in an at-risk population. From a public health standpoint, every $1 \mathrm{mmHg}$ reduction in the SBP could prevent approximately 10000 CHD deaths each year in the USA ${ }^{(3)}$. Moreover, a recent publication by Kishi et al. ${ }^{(52)}$, indicated that controlling $\mathrm{BP}$ in young adulthood has important implications for long-term heart health. Trends towards improvements in insulin and insulin sensitivity suggest that - but do not confirm - regular consumption of GSE beverages may offer metabolic benefits and warrant follow-up studies.

\section{Acknowledgements}

The authors are very grateful to Colin Kay from the University of East Anglia, UK, for kindly providing 5-phenylvaleric acid and syringic acid, Dirk M. Holstege and Paola Quifer-Rada from the Analytical Laboratory and Neil Willits, University of California Davis, for their technical assistance and the subjects who participated in this study.

This work was supported by Polyphenolics (grant number 2009-050).

The authors' contributions were as follows: B. B.-F. designed the research, reviewed data and interpreted results; I. E. and E. P. conducted the research and acquired data; I. E. performed laboratory analysis and interpretation; Y. Y. C. and A. W. performed phenolic analysis and interpretation; E. P. conducted statistical analyses and drafted the manuscript; B. B.-F. had primary responsibility for the final content of the manuscript. All the authors contributed to critical review and revision of the manuscript for important intellectual content. All authors read and approved the final version of the manuscript. The sponsor was not involved in designing the study, data analysis or interpretation of results.

None of the authors has any conflicts of interest.

\section{References}

1. Roger VL, Go AS, Lloyd-Jones DM, et al. (2012) Heart disease and stroke statistics - 2012 update: a report from the American Heart Association. Circulation 125, e2-e220.

2. Fields LE (2004) Mortality from stroke and ischemic heart disease increases exponentially with blood pressure. Hypertension 43, e28; author reply e28.

3. Lewington S, Clarke R, Qizilbash N, et al. (2002) Age-specific relevance of usual blood pressure to vascular mortality: a meta-analysis of individual data for one million adults in 61 prospective studies. Lancet 360, 1903-1913.

4. Vasan RS, Beiser A, Seshadri S, et al. (2002) Residual lifetime risk for developing hypertension in middle-aged women and men: ihe Framingham Heart Study. JAMA 287, 1003-1010.

5. Heidenreich PA, Trogdon JG, Khavjou OA, et al. (2011) Forecasting the future of cardiovascular disease in the United States: a policy statement from the American Heart Association. Circulation 123, 933-944.

6. Hansson L, Lindholm LH, Niskanen L, et al. (1999) Effect of angiotensin-converting-enzyme inhibition compared with conventional therapy on cardiovascular morbidity and mortality in hypertension: the Captopril Prevention Project (CAPPP) randomised trial. Lancet 353, 611-616.

7. Gaciong Z, Sinski M \& Lewandowski J (2013) Blood pressure control and primary prevention of stroke: summary of the recent clinical trial data and meta-analyses. Curr Hypertens Rep 15, 559-574.

8. Chobanian AV, Bakris GL, Black HR, et al. (2003) The Seventh Report of the Joint National Committee on Prevention, 
Detection, Evaluation, and Treatment of High Blood Pressure: the JNC 7 report. JAMA 289, 2560-2572.

9. Vasan RS, Larson MG, Leip EP, et al. (2001) Assessment of frequency of progression to hypertension in non-hypertensive participants in the Framingham Heart Study: a cohort study. Lancet 358, 1682-1686.

10. Eckel RH, Jakicic JM, Ard JD, et al. (2014) 2013 AHA/ACC guideline on lifestyle management to reduce cardiovascular risk: a report of the American College of Cardiology/American Heart Association Task Force on Practice Guidelines. J Am Coll Cardiol 63, 2960-2984.

11. James PA, Oparil S, Carter BL, et al. (2014) 2014 Evidencebased guideline for the management of high blood pressure in adults: report from the panel members appointed to the Eighth Joint National Committee (JNC 8). JAMA 311, 507-520.

12. Chong MF, Macdonald R \& Lovegrove JA (2010) Fruit polyphenols and CVD risk: a review of human intervention studies. Br J Nutr 104, Suppl. 3, S28-S39.

13. Shi J, Yu J, Pohorly JE, et al. (2003) Polyphenolics in grape seeds - biochemistry and functionality. J Med Food $\mathbf{6}$, 291-299.

14. Sivaprakasapillai B, Edirisinghe I, Randolph J, et al. (2009) Effect of grape seed extract on blood pressure in subjects with the metabolic syndrome. Metabolism 58, 1743-1746.

15. Ras RT, Zock PL, Zebregs YE, et al. (2013) Effect of polyphenol-rich grape seed extract on ambulatory blood pressure in subjects with pre- and stage I hypertension. Br J Nutr 110, 2234-2241.

16. Barona J, Aristizabal JC, Blesso CN, et al. (2012) Grape polyphenols reduce blood pressure and increase flow-mediated vasodilation in men with metabolic syndrome. $J$ Nutr $\mathbf{1 4 2}$, $1626-1632$

17. Robinson M, Lu B, Edirisinghe I, et al. (2012) Effect of grape seed extract on blood pressure in subjects with pre-hypertension. J Pharmacy Nutr Sci 2, 155-159.

18. Edirisinghe I, Burton-Freeman B \& Tissa Kappagoda C (2008) Mechanism of the endothelium-dependent relaxation evoked by a grape seed extract. Clin Sci (Lond) 114, 331-337.

19. Shen D, Wu Q, Wang M, et al. (2006) Determination of the predominant catechins in Acacia catechu by liquid chromatography/electrospray ionization-mass spectrometry. J Agric Food Chem 54, 3219-3224.

20. Singleton V \& Rossi J (1965) Colorimetry of total phenolics with phosphomolybdic-phosphotungstic acid reagents. Am J Enol Vitic 16, 144-158.

21. Alpert BS (2010) Validation of the Tiba Medical Ambulo 2400 ambulatory blood pressure monitor to the ISO Standard and BHS protocol. Blood Press Monit 15, 275-277.

22. Matthews DR, Hosker JP, Rudenski AS, et al. (1985) Homeostasis model assessment: insulin resistance and beta-cell function from fasting plasma glucose and insulin concentrations in man. Diabetologia 28, 412-419.

23. Corretti MC, Anderson TJ, Benjamin EJ, et al. (2002) Guidelines for the ultrasound assessment of endothelialdependent flow-mediated vasodilation of the brachial artery: a report of the International Brachial Artery Reactivity Task Force. J Am Coll Cardiol 39, 257-265.

24. Belcaro G, Ledda A, Hu S, et al. (2013) Grape seed procyanidins in pre- and mild hypertension: a registry study. Evid Based Complement Alternat Med 2013, 313142.

25. Clifton PM (2004) Effect of grape seed extract and quercetin on cardiovascular and endothelial parameters in high-risk subjects. J Biomed Biotechnol 2004, 272-278.

26. Ward NC, Hodgson JM, Croft KD, et al. (2005) The combination of vitamin $\mathrm{C}$ and grape-seed polyphenols increases blood pressure: a randomized, double-blind, placebo-controlled trial. J Hypertens 23, 427-434.

27. Sano A, Uchida R, Saito M, et al. (2007) Beneficial effects of grape seed extract on malondialdehyde-modified LDL. J Nutr Sci Vitaminol (Tokyo) 53, 174-182.

28. Urpi-Sarda M, Monagas M, Khan N, et al. (2009) Targeted metabolic profiling of phenolics in urine and plasma after regular consumption of cocoa by liquid chromatographytandem mass spectrometry. J Chromatogr A 1216, 7258-7267.

29. Koli R, Erlund I, Jula A, et al. (2010) Bioavailability of various polyphenols from a diet containing moderate amounts of berries. J Agric Food Chem 58, 3927-3932

30. Mountzouris KC, McCartney AL \& Gibson GR (2002) Intestinal microflora of human infants and current trends for its nutritional modulation. Br J Nutr 87, 405-420.

31. Benson AK, Kelly SA, Legge R, et al. (2010) Individuality in gut microbiota composition is a complex polygenic trait shaped by multiple environmental and host genetic factors. Proc Natl Acad Sci U S A 107, 18933-18938.

32. Rechner AR, Spencer JP, Kuhnle G, et al. (2001) Novel biomarkers of the metabolism of caffeic acid derivatives in vivo. Free Radic Biol Med 30, 1213-1222.

33. Natsume M, Osakabe N, Yamagishi M, et al. (2000) Analyses of polyphenols in cacao liquor, cocoa, and chocolate by normal-phase and reversed-phase HPLC. Biosci Biotechnol Biochem 64, 2581-2587.

34. Schroeter H, Heiss C, Balzer J, et al. (2006) (-)-Epicatechin mediates beneficial effects of flavanol-rich cocoa on vascular function in humans. Proc Natl Acad Sci U S A 103, 1024-1029.

35. Baba S, Osakabe N, Natsume M, et al. (2001) Absorption and urinary excretion of (-)-epicatechin after administration of different levels of cocoa powder or (-)-epicatechin in rats. J Agric Food Chem 49, 6050-6056.

36. Kar P, Laight D, Rooprai HK, et al. (2009) Effects of grape seed extract in type 2 diabetic subjects at high cardiovascular risk: a double blind randomized placebo controlled trial examining metabolic markers, vascular tone, inflammation, oxidative stress and insulin sensitivity. Diabet Med 26, 526-531.

37. Engler MB, Engler MM, Chen CY, et al. (2004) Flavonoid-rich dark chocolate improves endothelial function and increases plasma epicatechin concentrations in healthy adults. $J$ Am Coll Nutr 23, 197-204.

38. Heiss C, Kleinbongard P, Dejam A, et al. (2005) Acute consumption of flavanol-rich cocoa and the reversal of endothelial dysfunction in smokers. $\mathrm{J} \mathrm{Am}$ Coll Cardiol $\mathbf{4 6}$, 1276-1283.

39. Ottaviani JI, Actis-Goretta L, Villordo JJ, et al. (2006) Procyanidin structure defines the extent and specificity of angiotensin I converting enzyme inhibition. Biochimie 88, 359-365.

40. Alvarez E, Rodino-Janeiro BK, Jerez M, et al. (2012) Procyanidins from grape pomace are suitable inhibitors of human endothelial NADPH oxidase. J Cell Biochem 113, 1386-1396.

41. Addison S, Stas S, Hayden MR, et al. (2008) Insulin resistance and blood pressure. Curr Hypertens Rep 10, 319-325.

42. Hu FB \& Stampfer MJ (2005) Insulin resistance and hypertension: the chicken-egg question revisited. Circulation 112, 1678-1680.

43. Kim JA, Montagnani M, Koh KK, et al. (2006) Reciprocal relationships between insulin resistance and endothelial dysfunction: molecular and pathophysiological mechanisms. Circulation 113, 1888-1904.

44. Rodriguez-Mateos A, Rendeiro C, Bergillos-Meca T, et al. (2013) Intake and time dependence of blueberry flavonoidinduced improvements in vascular function: a randomized, controlled, double-blind, crossover intervention study with 
mechanistic insights into biological activity. Am J Clin Nutr 98 1179-1191.

45. Sowers JR (2004) Insulin resistance and hypertension. Am J Physiol Heart Circ Physiol 286, H1597-H1602.

46. El-Atat F, Aneja A, McFarlane S, et al. (2003) Obesity and hypertension. Endocrinol Metab Clin North Am 32, 823-854.

47. Goff DC Jr, Zaccaro DJ, Haffner SM, et al.2003) Insulin sensitivity and the risk of incident hypertension: insights from the Insulin Resistance Atherosclerosis Study. Diabetes Care 26, 805-809.

48. Forman JP, Choi H \& Curhan GC (2009) Uric acid and insulin sensitivity and risk of incident hypertension. Arch Intern Med 169, 155-162.

49. Edirisinghe I, Randolph J, Cheema M, et al. (2012) Effect of grape seed extract on postprandial oxidative status and metabolic responses in men and women with the metabolic syndrome. Funct Food Health Dis 2, 508-521.

50. Meeprom A, Sompong W, Suwannaphet W, et al. (2011) Grape seed extract supplementation prevents high-fructose diet-induced insulin resistance in rats by improving insulin and adiponectin signalling pathways. Br J Nutr 106, 1173-1181.

51. Grassi D, Desideri G, Necozione S, et al. (2008) Blood pressure is reduced and insulin sensitivity increased in glucose-intolerant, hypertensive subjects after 15 days of consuming high-polyphenol dark chocolate. J Nutr 138, 1671-1676.

52. Kishi S, Teixido-Tura G, Ning H, et al. (2015) Cumulative blood pressure in early adulthood and cardiac dysfunction in middle age: the CARDIA study. J Am Coll Cardiol 65, 2679-2687. 